02

\title{
Плотность состояний тонких сверхпроводящих каналов в режиме квантовых флуктуаций параметра порядка
}

\author{
(С) К.Ю. Арутюнов ${ }^{1,2}$, J.S. Lehtinen ${ }^{3}$, A.A. Радкевич4,5, А.Г. Семенов ${ }^{4,5}$, А.Д. Заикин ${ }^{4,6}$ \\ ${ }^{1}$ Национальный исследовательский университет „Высшая школа экономики“, \\ Московский институт электроники и математики, \\ Москва, Россия \\ ${ }^{2}$ Институт физических проблем РАН им. П.Л. Капицы, \\ Москва, Россия \\ ${ }^{3}$ VTT Technical Research Centre of Finland Ltd., Centre for Metrology MIKES, \\ P.O. Box 1000, FI-02044 VTT, Finland \\ ${ }^{4}$ Отделение теоретической фризики им. И.Е. Тамма, Физический институт им. П.Н. Лебедева РАН, \\ Москва, Россия \\ ${ }^{5}$ Московский ффизико-технический институт, \\ Долгопрудный, Россия \\ ${ }^{6}$ Institute of Nanotechnology, Karlsruhe Institute of Technology, \\ Karlsruhe, Germany \\ E-mail: karutyunov@hse.ru
}

\begin{abstract}
Экспериментально исследовались вольт-амперные характеристики туннельных контактов сверхпроводник-изолятор-сверхпроводник $(\mathrm{C} 1-И-\mathrm{C} 2)$, где сверхпроводящий электрод $\mathrm{C} 2$ представлял из себя тонкий нанопровод. Обнаруженное размытие щелевых особенностей интерпретируется как проявление эффекта квантовых флуктуаций параметра порядка. Предложена модель, учитывающая уширение плотности состояний за счет взаимодействия электронов с плазмонной модой Муи-Шёна, возникающей в квазиодномерном сверхпроводящем канале в режиме квантовых флуктуаций параметра порядка. Модель дает разумное качественное согласие с экспериментом.
\end{abstract}

DOI: 10.21883/FTT.2017.11.45042.01k

\section{1. Введение}

В последнее время проявился интерес к изучению квазиодномерной сверхпроводимости [1]. Спецификой таких систем является яркое проявление флуктуационных эффектов, которые влияют как на транспортные [2], так и - термодинамические свойства [3]. При рассмотрении флуктуаций сверхпроводящего параметра порядка $\Delta=|\Delta| e^{i \phi}$ необходимо различать флуктуации фазы $\phi$ и модуля $|\Delta|$. В частности, было показано [4], что в тонких сверхпрводящих каналах флуктуации фазы с неизбежностью приводят к возбуждению „мягкой“ звукоподобной волны зарядовой плотности - т. н. моды Муи-Шёна [5]. Взаимодействие носителей заряда с такими плазмонами приводит к перераспределению электронных состояний, в частности - к размытию сингулярности плотности состояний в области сверхпроводящей щели $\Delta$. Целью работы являлось экспериментальное и теоретическое исследование этого интригующего явления.

\section{2. Методика эксперимента}

Наноструктуры изготовлялись методом взрывной электроннолучевой литографии и направленного вакуумного напыления. Было изготовлено несколько многотерминальных туннельных С1-И-С2 структур, где сверхпроводящий контакт $\mathrm{C} 1$ - „массивный“ алюминий, изолятор И - тонкий слой оксида алюминия, а электроды С2 представляли из себя тонкие титановые нанопровода (рис. 1). Предварительные исследования показали, что в титановых каналах при эффективных диаметрах (корень из сечения) менее $40 \mathrm{~nm}$ форма сверхпроводящего перехода $R(T)$ сильно уширена за счет вклада квантовых флуктуаций фазы параметра порядка - квантовых проскальзываний фазы [6]. Структуры, изученные в настоящей работе, были изготовлены таким образом, чтоб диаметр тонких нанопроводов С2 находился именно в той области, где квантовые флуктуации должны отчетливо проявляться [6]. Измерения проводились в области сверхнизких температур, мно-

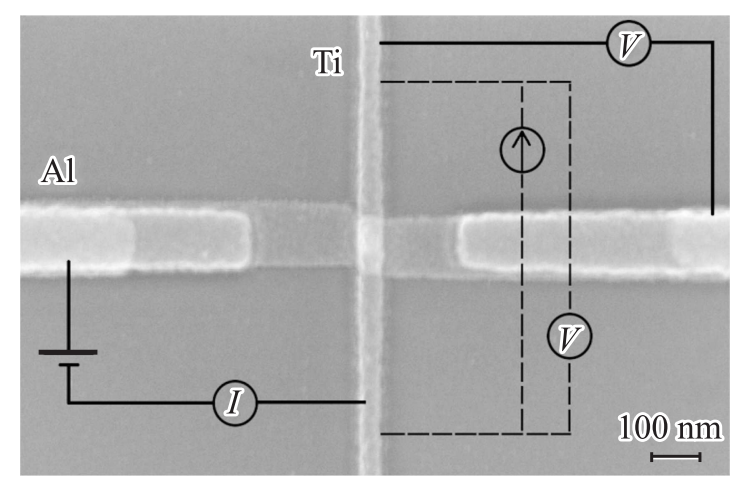

Рис. 1. Электронная микрофотография структуры и схема измерений. 


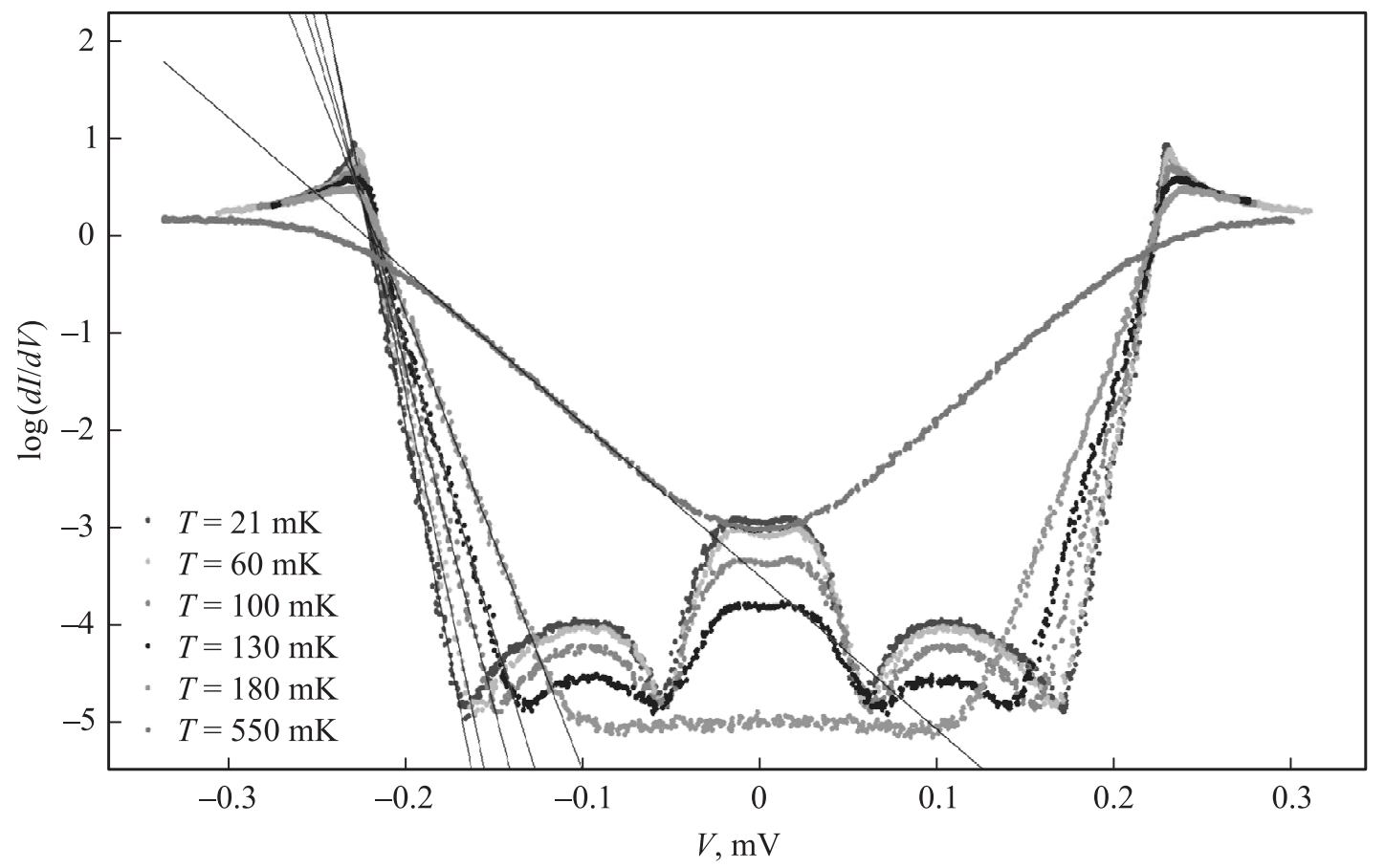

Рис. 2. Дифференциальные ВАХ при различных температурах для $\mathrm{C} 1-$ И-С2 структуры, где сверхпроводящий контакт С1 „массивный“ алюминий, И - оксид алюминия, электроды С2 - тонкий титановый нанопровод с диаметром порядка $30 \mathrm{~nm}$. Следует обратить внимание, что ось ординат построена в логарифмическом масшабе, демонстрирующим эффект (сплошные линии) экспоненциального „пролезания“ плотности состояний в область подщелевых значений энергий. Природа слабых (отчетливо видных лишь в логарифмическом масштабе) немонотонных особенностей в области $e V \ll\left|\Delta_{1}\right|+\left|\Delta_{2}\right|$ не вполне ясна и выходит за рамки обсуждений настоящей работы.

го меньших критических температур как алюминия $\left(T_{c} \sim 1.4 \mathrm{~K}\right)$, так и титана $\left(T_{c} \sim 0.4 \mathrm{~K}\right)$. Рабочей гипотезой было предположение, что за счет квантовых флуктуаций параметра порядка, вольт-амперные характеристики (BAX) будут размываться в области щелевых смещений $e V=\left|\Delta_{1}\right|+\left|\Delta_{2}\right|$.

\section{3. Результаты и обсуждение}

В рамках классической модели БКШ температурная зависимость плотности состояний обусловлена лишь соответствующим подавлением щели $\Delta$, а тепловое размытие полностью отсутствует, что формально приводит к бесконечно резкой $\mathrm{BAX} \mathrm{C} 1-И-\mathrm{C} 2$ контакта в области смещений $e V=\left|\Delta_{1}\right|+\left|\Delta_{2}\right|$ [7]. В реальных экспериментах всегда наблюдается конечное размытие щелевых особенностей BAX, которое обычно приписывается двум вкладам: (a) различные инструментальные эффекты (электромагний шум, ненулевое время интегрирования и т.д.) и (b) конечное время жизни квазичастичных возмущений, которое формально определяется феноменологическим параметром Дайнса $Г$

$$
N(E) \approx N\left(E_{\mathrm{F}}\right) \operatorname{Re}\left(\frac{E+i \Gamma}{\sqrt{(E+i \Gamma)^{2}-\Delta^{2}}}\right),
$$

где $N\left(E_{\mathrm{F}}\right)$ - плотность состояний на уровне Ферми.
При низких температурах $k_{\mathrm{B}}(T) \ll \Delta_{1,2}$ для туннельных $\mathrm{C} 1-$ И-С2 контактов мезоскопических размеров в эксперименте реально получить достаточно резкие ВАХ, соответствующие плотности состояния сверхпроводника (1) с величиной параметра Дайнса Г/ $\Delta_{1,2}<10^{-3}[8]$.

$\mathrm{B}$ наших измерениях $\mathrm{BAX} \mathrm{C} 1-И-\mathrm{C} 2$ структур с эффективным диаметром титанового электрода С2 более $40 \mathrm{~nm}$ продемонстрировали типичные зависимости для массивных сверхпроводников: резкая щелевая особенность при смещениях $e V=\left|\Delta_{1}\right|+\left|\Delta_{2}\right|$. С уменьшением диаметра титанового контакта С2 форма ВАХ качественным образом меняется. Пример дифференциальной ВАХ приведен на рис. 2. Прослеживаются два эффекта: чем меньше диаметр нанопровода С2, тем $(a)$ меньше сверхпроводящая щель титана $\Delta_{2}$ и $(b)$ тем больше размытие щелевой особенности. Причина первого явления может быть связана с предсказанным ранее подавлением величины параметра порядка квантовыми флуктуациями [9]. Величина данного эффекта контролируется безразмерной обратной нормальной проводимостью $1 / g$ сегмента проволоки размером равным сверхпроводящей длине когерентности. При уменьшении диаметра проволоки $1 / g$ растет. Следовательно, подавление параметра порядка усиливается [9], что и наблюдается на эксперименте.

Здесь более подробно рассмотрим второй эффект размытие щелевой особенности ВАХ. Данный эффект 


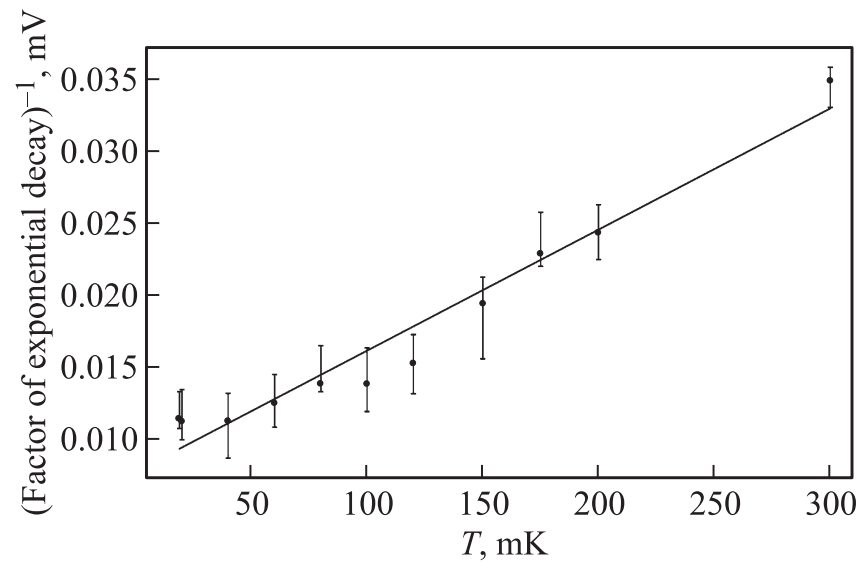

Рис. 3. Температурная зависимость декремента затухания плотности состояний в подщелевой области энергий $E \delta \leq\left|\Delta_{1}\right|+\left|\Delta_{2}\right|$. Фитирование экспериментальной зависимости прямой линией дает наклон $8.4 \cdot 10^{-5} \mathrm{~V} / \mathrm{K}$, что в пределах погрешности совпадает с теоретически предсказанным значением $k_{\mathrm{B}} / e \approx 8.6 \cdot 10^{-5} \mathrm{~V} / \mathrm{K}$.

интерпретируется нами в рамках недавно предложенной модели [10], учитывающей квантовые флуктуации фазы параметра порядка. Подобные флуктуации в низкоразмерных сверхпроводниках с неизбежностью приводят к возбуждению „мягкой“ звукоподобной волны зарядовой плотности моды Муи-Шёна [5]. Взаимодействие носителей заряда с таким плазмонами приводит к перераспределению электронных состояний, в частности - к размытию сингулярности плотности состояний в области сверхпрводящей щели $\Delta$. Моды Муи-Шёна можно описать эффективным действием $[1,4]$

$$
S[\phi]=\frac{C}{8 e^{2}} \int\left[\phi^{2}-v^{2}\left(\partial_{x} \phi\right)^{2}\right] d t d x,
$$

где $C$ - удельная емкость провода на единицу длины, $e$ - заряд электрона, а $v \approx\left(\pi \Delta \sigma_{N} s / C\right)^{1 / 2}-$ скорость волны Муи-Шёна, $\sigma_{N}=e^{2} D N\left(E_{\mathrm{F}}\right)-$ проводимость канала с сечением $s$ в нормальном состоянии, $D-$ коэффициент диффузии.

Усредняя функции Грина по действию (2) можно получить выражение для плотности состояний квазиодномерного сверхпроводника в режиме распространения плазмонных волн Муи-Шёна $N_{\mathrm{M}-\mathrm{S}}(E)$, формально совпадающее с выражением (1) с конечной величиной размытия Г. В режиме слабых флуктуаций $\alpha \ll 1$ при низких температурах $k_{\mathrm{B}} T \ll \Delta$ расчет [10] дает специфическую зависимомость параметра Дайнса $\Gamma \sim \alpha T$, где $\alpha=\left(e^{2} / 2 \pi(C v)\right.$ - безразмерная величина, характеризующая „силу“ флуктуаций. Очевидно, что в этом случае размытие плотности состояния становится зависимым от сечения провода $s$, проводимости материала в нормальном состоянии $\sigma_{N}$, величины сверхпроводящей щели $\Delta_{2}$ и температуры $T$.

Использование стандартного выражения для формы BAX туннельного С1-И-С2 контакта [7], дает качественно удовлетворительное согласие с экспериментом. Например, четко прослеживается экспоненциальное „пролезание“ плотности состояний в подщелевую область (рис. 2), что совпадает с предсказанием модели $N_{\mathrm{M}-\mathrm{S}}(E) \sim \exp \left[\left(E-\Delta_{2}\right) / k_{\mathrm{B}} T\right]$. Температурная зависимость декремента затухания с хорошей точностью определяется теоретическим значением $k_{\mathrm{B}} / e=8.6 \cdot 10^{-5} \mathrm{~V} / \mathrm{K}$ (рис. 3 ).

\section{4. Заключение}

Были исследованы туннельные ВАХ C1-И-С2 контактов, где один из электродов - тонкий сверхпроводящий канал в режиме квантовых флуктуаций параметра порядка. Предложенная модель дает правильное описание формы таких ВАХ, однако полное количественное сравнение экспериментальных результатов с предсказаниями теории можно будет провести лишь после учета не только размытия плотности состояний за счет возбуждения плазмонных мод [10], но и „прямого“ влияния квантовых флуктуаций параметра порядка [9] на электронную плотность состояния $N(E)$. Теоретическое описание такого влияния составит предмет отдельного исследования.

В работе использованы материалы проекта Т3-89 „Квантовые кооперативные явления при низких и сверхнизких температурах“ в рамках программы „Научный фонд Национального исследовательского университета “Высшая школа экономики,, (НИУ ВШЭ) и с использованием средств субсидии на государственную поддержку ведущих университетов Российской Федерации в целях повышения их конкурентоспособности среди ведущих мировых научно-образовательных центров, выделенной НИУ ВШЭ.

\section{Список литературы}

[1] K.Yu. Arutyunov, D.S. Golubev, A.D. Zaikin. Phys. Rep. 464, 1 (2008).

[2] N. Giordano. Phys. Rev. Lett. 61, 2137 (1988).

[3] K.Yu. Arutyunov, T.T. Hongisto, J.S. Lehtinen, L. Leino, A. Vasiliev. Sci. Rep. 2, 213 (2012).

[4] A. van Otterlo, D.S. Golubev, A.D. Zaikin, G. Blatter. Eur. Phys. J. B 10, 131 (1999).

[5] J.E. Mooij, G. Schön. Phys. Rev. Lett. 55, 114 (1985).

[6] J.S. Lehtinen, K. Zakharov, K.Yu. Arutyunov. Phys. Rev. B 85, 094508 (2012).

[7] M. Tinkham. Introduction to superconductivity. $2 \mathrm{~d}$ ed. McGraw-Hill, Inc., N. Y. (1996). 454 p.

[8] F. Giazotto, T.T. Heikkilä, A. Luukanen, A.M. Savin, J.P. Pekola. Rev. Mod. Phys. 78, 217 (2006).

[9] D.S. Golubev, A.D. Zaikin. Phys. Rev. B 78, 144502 (2008).

[10] A.A. Radkevich, A.G. Semenov, A.D. Zaikin. Phys. Rev. (2017). In press. 